\title{
Análisis económico de sistemas productivos ovinos de Córdoba, Argentina. Estudio de casos
}

\author{
Freire, V.; D. Agüero, M. Ponce Crivellaro, M. Vigliocco y G. Sandoval
}

\begin{abstract}
RESUMEN
En un contexto de gran heterogeneidad organizativa y de recursos se estimaron los resultados económicos de la producción ovina de Córdoba, Argentina, con el propósito de generar referencias para la toma de decisiones relacionadas con el desarrollo del sector. Mediante estudio de casos se recogió información en seis agro-regiones, a través de encuestas semiestructuradas y entrevistas en profundidad. La venta de cordero fue el rubro de mayor participación entre los ingresos ( $83 \%$ de los casos); mano de obra (41\%) y suplementos $(17 \%)$, los principales componentes en el promedio de costos totales. La actividad ovina logró márgenes brutos positivos en pequeños y medianos establecimientos serranos y de llanura, extensivos y semi-intensivos, especializados o diversificados. Estrategias técnicas, comerciales y de gestión, permitieron alcanzar márgenes brutos similares o superiores a los de ganadería vacuna. Factores climáticos, técnicos y estructurales generaron márgenes netos negativos en establecimientos del noroeste y centro-oeste de Córdoba. Se concluye que si bien la producción ovina acrecentó el beneficio económico en la mitad de los sistemas analizados, de no mediar cambios de estrategias productivas y de políticas públicas vinculadas al sector, la viabilidad a mediano plazo se verá afectada en los sistemas más vulnerables.
\end{abstract}

Palabras clave: resultados económicos, eficiencia económica, estrategias productivas, regiones agroeconómicas homogéneas.

Freire, V.; D. Agüero, M. Ponce Crivellaro, M. Vigliocco and G. Sandoval, 2013. Economic analysis of productive systems sheep of Cordoba, Argentina. Case study. Agriscientia 30 (1): 37-47

\section{SUMMARY}

In a context of great organizational heterogeneity and resources raises estimate the economic results of the sheep production of Cordoba, Argentina, with the purpose to generate references for decision-making related to the development of the sector. On the basis of cases study information was collected in six agroregions, through semi-structured surveys and in-depth interviews. The sale of lamb was the largest participation between income (83\% of cases); labor (41 $\%)$ and supplements $(17 \%)$ the main components in the average total cost. 
The ovine activity achievement positive gross margins in small and mediumsized establishments highland and lowland, extensive and semi-intensive, specialized or diversified. Strategies, techniques, business and management, enabled to achieve gross margins similar or superior to those of beef cattle. Climatic, technical and structural factors generated margins net negative in establishments of northwest and center-west of Cordoba. It is concluded that while the sheep production increased the economic benefit in half of the systems analyzed, without changes related to productive strategies and public policy the sector, the medium-term viability will be affected in the most vulnerable systems.

Key words: economic results, economic efficiency, productive strategies, homogeneous agro - economic regions

V. Freire, D. Agüero, M. Ponce Crivellaro, M. Vigliocco y G. Sandoval: Dpto. Economía Agraria, Fac. de Agronomía y Veterinaria, Universidad Nacional de Río Cuarto. Ruta 36, km 601. 5800 Río Cuarto, Argentina. Correspondencia a: vfreire@ayv.unrc.edu.ar

\section{INTRODUCCIÓN}

La evolución de la ganadería ovina de la provincia de Córdoba ha seguido la tendencia observada a nivel nacional, con un marcado descenso de las existencias hasta el año 2002 (147 mil cabezas), para recuperarlas en 2011 (223 mil cabezas) (MAGyP, 2011). El sector atraviesa un período favorable para su recuperación, impulsado, entre otros factores, por el apoyo de la Ley 25.422, de Recuperación de la Ganadería Ovina (Arzubi et al., 2011)

Mc Cormick (2003) planteó que en el sector ovino existe una combinación de problemas que requieren intervenciones de tipo "sistémico particular", y destacó para el sector primario falencias productivas, comerciales y escasa capacidad de gestión.

En la provincia de Córdoba, el 95\% de los establecimientos agropecuarios (EAP) ovinos trabaja menos de 100 cabezas y concentra casi el 65\% de las existencias (MAGyP, 2011). La producción -inserta en un sector de alta diversidad productiva- es heterogénea, tanto en la organización como en la dotación de recursos. Escala productiva y dificultades impositivas derivan en la comercialización informal en la mayoría de los casos (Agüero et al., 2010).

En la región pampeana, por el costo de oportunidad de la tierra, el ovino ocupa un lugar secundario. Los sistemas predominantemente extensivos, con majadas de 50 a 150 ovejas, tienen una receptividad de 2 a 6 ovejas por hectárea (Rodríguez et al., 2010). Conjuntamente con vacunos, posibilitan mayor nivel de producción que el logrado con las especies por separado, y tienen efectos positivos sobre la eficacia de uso de las pasturas (IDIAXXI, 2004).

Santamaría et al. (1998) sostienen que los sistemas ovinos pastoriles pueden ser rentables, dependiendo tanto de los costos de producción, fundamentalmente alimentación, como de la capacidad para mejorar los resultados técnicos sin aumento de costos. En sistemas agriculturizados, Vigliocco et al. (2011) mencionaron que los costos de mano de obra, alimentación y suplementación fueron los más importantes. Arzubi et al. (2012) expresaron que los modelos carniceros de la Cuenca del Salado Norte y mixtos de Mar y Sierras fueron los más competitivos. Rodríguez et al. (2011) plantearon que el sistema de cría vacuna y ovina tuvo mayor estabilidad de resultados económicos ante cambios de precios y variables técnicas que el sistema vacuno puro.

Issaly et al. (2010) refirieron que el "productor tradicional conservador" no introduce cambios técnicos, ni toma créditos. La producción asume fines comerciales y de autoconsumo que justifican su mantenimiento aunque el aporte económico sea reducido. Para los autores, este tipo de productor ovino tiende a desaparecer. En contraposición, el "productor innovador" asume estrategias de diversificación, está abierto a cambios y a la toma de créditos, manteniéndose en la actividad por sus expectativas favorables respecto a los mercados.

Estudios económicos realizados en diferentes regiones de Córdoba mostraron márgenes brutos positivos para la actividad ovina. En algunas regiones los márgenes netos fueron negativos debido a 
factores ambientales, económicos, organizacionales y comerciales (Freire et al., 2011a).

El objetivo de este trabajo fue estimar y analizar los indicadores económicos de sistemas ovinos de diferentes agro-regiones de Córdoba, que resultan de la combinación de recursos y estrategias productivas diversas, con el propósito de generar referencias para la toma de decisiones relacionadas con el desarrollo del sector.

\section{MATERIALES Y MÉTODOS}

La heterogeneidad respecto a características ecológicas, recursos y combinaciones técnico-productivas -entre otros factores- conduce a abordar aquellos sistemas que representen la mayor diversidad posible. La economía de la empresa requiere de metodologías de investigación capaces de recoger la complejidad existente, combinando información cualitativa y cuantitativa, subjetiva y objetiva, interna y externa al propio fenómeno estudiado (Villareal Larrinaga \& Landeta Rodríguez, 2009).

Para Yin (1994) un estudio de casos es "una investigación empírica que investiga un fenómeno contemporáneo en su contexto real, donde los límites entre el fenómeno y el contexto no se muestran de forma precisa, y en el que múltiples fuentes de evidencia son usadas". El estudio de múltiples casos permite reforzar la validez interna y externa, reproduciendo la experiencia de cada caso en los otros (Villareal Larrinaga \& Landeta Rodríguez, 2009).

La selección de casos se realizó con el enfoque de región agroproductiva (Tsakougmagkos et al., 2000), tomando como principal criterio la proporción de existencias ovinas respecto al total provincial. En primer término se identificaron los departamentos de Córdoba que concentraban las mayores existencias (SENASA, 2008); luego, las zonas agroeconómicas homogéneas (ZAH) en los que éstos estaban contenidos, entendiendo que dichas regiones mantienen cierta homogeneidad en sus características ambientales, estructurales, socio-económicas, productivas e institucionales (Guida Daza \& Sánchez, 2009). Las existencias ovinas de las seis ZAH seleccionadas concentraban el 75,8\% del total provincial, según las estadísticas censales publicadas. En la Tabla 1 se destacan sus principales características agroecológicas y técnico-productivas.

A partir de la revisión de la literatura específica, información de estudios previos (Freire et al., 2009, 2010; Agüero et al., 2010), informantes calificados y referentes técnicos, se seleccionó un establecimiento agropecuario (EAP) en cada ZAH. Se tomó en cuenta que la producción ovina estuviera fundamentalmente destinada al mercado, por su perfil más innovador y su continuidad en el tiempo (Issaly et al., 2010). Los casos 1 y 2 correspondieron a las ZAH de Cruz del Eje y Cosquín, región de monte árido (Tsakougmagkos et al., 2000). Los casos 3 a 6 se ubicaron en las ZAH Río Cuarto, Villa María, Laboulaye y Villa Huidobro, correspondientes a la región pampeana.

Para su estudio se elaboraron registros de recolección de datos técnicos, económicos y factores productivos, recabados mediante encuestas realizadas a los productores. Complementariamente, se desarrollaron entrevistas en profundidad para obtener datos de estrategias productivas, financieras y comerciales adoptadas en dichos sistemas.

Se calcularon márgenes brutos (MB) de la actividad ovina por diferencia entre ingresos brutos (IB) y costos directos (CD) (Peretti et al., 2006). A los $\mathrm{MB}$ se les descontaron los costos indirectos $(\mathrm{Cl})$, para obtener así los márgenes netos (MN). Debido a que los sistemas evaluados compartían gastos de estructura, infraestructura y equipamiento

Tabla 1. Zonas agroecológicas y existencias ovinas de Córdoba.

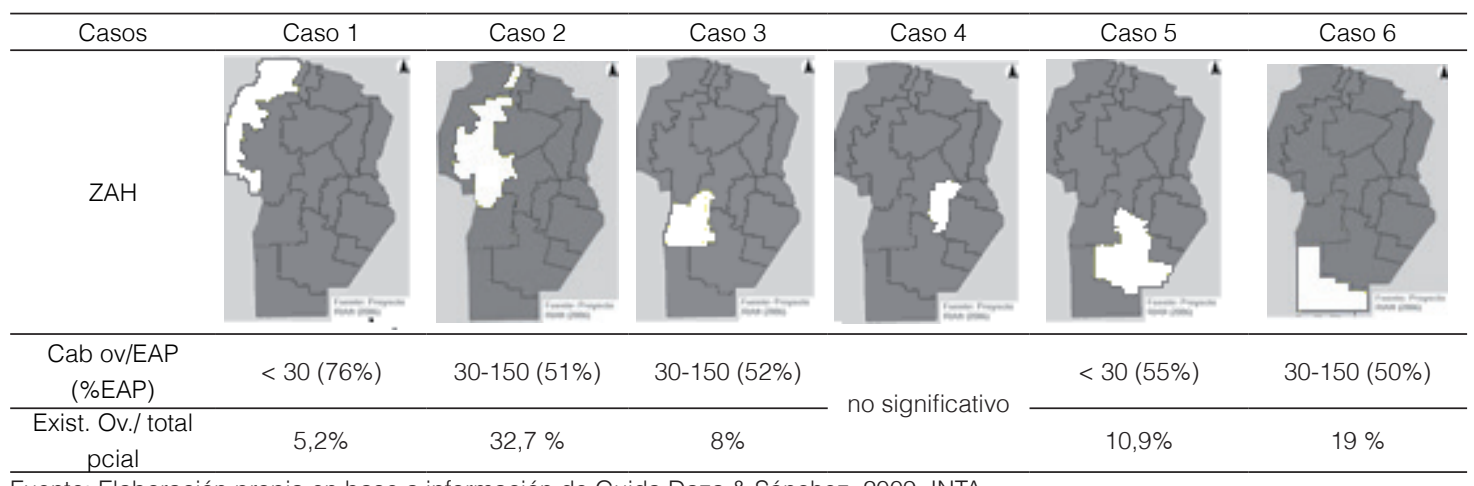

Fuente: Elaboración propia en base a información de Guida Daza \& Sánchez, 2009. INTA. 
general con diversas actividades productivas, los $\mathrm{Cl}$ se integraron con los valores proporcionales de costos de mano de obra y amortizaciones de mejoras y equipos atribuidos a la actividad ovina (Frank, 1985). La suma de CD y Cl permitió calcular los costos totales (CT) de la actividad ovina. Se calcularon medidas de eficiencia económica relacionando la ganancia obtenida, parcial (MB) y total (MN), por unidad de recursos utilizados (ha, CD y CT) y número de corderos logrados. Se tomaron precios de insumos y productos suministrados por los propietarios entrevistados para los ciclos 2008/2009 (cinco casos) y 2009/2010 (un caso), actualizados a moneda de junio 2011, mediante el Sistema de Índices de Precios Mayoristas (INDEC, 2012).

\section{Descripción de los casos estudiados}

\section{Caso 1}

El establecimiento ubicado en zona de chacras hortícolas del departamento Cruz del Eje cuenta con una superficie de 44 ha propias, parte cedida en arrendamiento y 13 ha de superficie ocupadas por una represa y canales de regadío. La actividad ovina está orientada hacia la producción de carne para aprovechar la zona turística cercana. Aunque el "nuevo productor rural" proyecta crecer a mediano o largo plazo, su pequeña majada, según el criterio señalado por Arzubi et al. (2011), no alcanzaba el tamaño comercial mínimo de 100 ovejas (Tabla 2). El período de sequía le ocasionó considerables pérdidas, y una diferencia de inventario negativa $(-18 \%$ del IB).

\section{Caso 2}

El establecimiento mixto del departamento Punilla cuenta con aproximadamente 1500 ha propias de pastizales de altura en el monte serrano. El emprendimiento ovino, de escasa antigüedad, se desarrolló para usufructuar la ventaja turística de la serranía cordobesa. Comercializa la lana con valor agregado, al participar en los programas "Asistencia para el mejoramiento de la calidad de la lana" (Prolana) y "Cadenas de Valor", del INTA e INTI, respectivamente. La gran dificultad para controlar a los predadores naturales de la región (pumas) y la mortandad de dos carneros por ingestión de plantas tóxicas, condujeron a un reducido porcentaje de señalada (Tabla 4).

\section{Caso 3}

Cercano a la ciudad de Río Cuarto, en el departamento homónimo, este sistema arrienda el casco de un establecimiento (5 ha) que le brinda vivienda al grupo familiar, galpones y corrales a la producción lechera caprina. Realiza trueque de corderos para utilizar los rastrojos de cultivos agrícolas linderos. Las crías ovina y caprina y sus subproductos artesanales sustentan a la familia. Los productores (matrimonio) organizan dos pariciones al año para obtener una oferta de corderos de mayor continuidad. Las mayores pérdidas del ciclo $(61 \%)$ se debieron a abigeato (Tabla 4$)$. Por la dificultad de determinar la incidencia económica en la actividad ovina primaria, no fueron considerados los costos de arrendamiento, ni la venta de cueros y fiambres ovinos.

\section{Caso 4}

El sistema del departamento San Martín se incluyó en el análisis por considerarlo una alternativa viable para pequeños establecimientos, a pesar de las escasas existencias ovinas de la ZAH. La sucesión familiar, con tradición ovina generacional, posee 41 ha y orienta sus estrategias hacia la diversificación de actividades (Tabla 3). La adquisición de reproductores se les dificultó, dado que los precios de mercado fueron superiores a los establecidos por la Ley Ovina. Su escala les representó una limitante para acceder a más créditos de inversión y mejorar la infraestructura.

Tabla 2. Variables estructurales de los sistemas ovinos de Córdoba

\begin{tabular}{|c|c|c|c|c|c|c|c|}
\hline \multicolumn{2}{|l|}{ Descripción } & Caso 1 & Caso 2 & Caso 3 & Caso 4 & Caso 5 & Caso 6 \\
\hline \multicolumn{2}{|c|}{ Período analizado } & 2008/2009 & 2008/2009 & 2008/2009 & 2008/2009 & 2008/2009 & $2009 / 2010$ \\
\hline \multirow{5}{*}{$\begin{array}{l}\text { Disponibilidad } \\
\text { de factores } \\
\text { productivos } \\
\text { destinados a } \\
\text { la producción } \\
\text { ovina }^{(1)}\end{array}$} & Tierra & Insuficiente & Insuficiente & No posee & Suficiente & Suficiente & Suficiente \\
\hline & Capital M y $E^{(2)}(\$)$ & $6.997,3$ & $12.431,3$ & $15.591,6$ & $30.199,7$ & $61.559,6$ & $98.011,3$ \\
\hline & Capital M y $E^{(2)}$ (\$/oveja) & 106,0 & 96,4 & 106,8 & 328,3 & 252,3 & 765,7 \\
\hline & Capital ovejas (cabezas) & 66 & 129 & 146 & 92 & 244 & 128 \\
\hline & Trabajo (EH/año) & 0,150 & 0,200 & 0,500 & 0,125 & 0,500 & 0,120 \\
\hline \multicolumn{2}{|c|}{ Organización social del trabajo } & Asalariado & Asalariado & Familiar & Familiar & Fam/Asalar & Asalar/Fam \\
\hline
\end{tabular}

Fuente: Elaboración propia

Referencias: (1) Disponibilidad relativa (Caracciolo de Basco et al., 1981); (2) M: mejoras; E: equipos; valores monetarios expresados en moneda constante de junio/2011. 
Tabla 3. Principales estrategias de los sistemas ovinos de Córdoba

\begin{tabular}{|c|c|c|c|c|c|c|c|}
\hline \multicolumn{2}{|l|}{ Descripción } & Caso 1 & Caso 2 & Caso 3 & Caso 4 & Caso 5 & Caso 6 \\
\hline \multicolumn{2}{|c|}{$\begin{array}{l}\text { Principales actividades del } \\
\text { EAP }\end{array}$} & $\begin{array}{c}\text { Fardos de } \\
\text { alfalfa }\end{array}$ & Cría vacuna & $\begin{array}{l}\text { Ovinos y } \\
\text { caprinos }\end{array}$ & $\begin{array}{c}\text { Agricultura - } \\
\text { porcinos }\end{array}$ & Agricultura & $\begin{array}{c}\text { Invernada } \\
\text { vacuna }\end{array}$ \\
\hline \multicolumn{2}{|c|}{$\begin{array}{l}\text { Actividad/es } \\
\text { complementaria/s del EAP }\end{array}$} & $\begin{array}{l}\text { Olivos - } \\
\text { Ovinos }\end{array}$ & $\begin{array}{l}\text { Ovinos - } \\
\text { agricultura }\end{array}$ & $\begin{array}{c}\text { subproductos } \\
\text { caprinos y } \\
\text { ovinos }\end{array}$ & $\begin{array}{c}\text { Ovinos } \\
\text {-hotelería } \\
\text { equina } \\
\end{array}$ & Ovinos & $\begin{array}{l}\text { Ovinos } \\
\text {-agricultura }\end{array}$ \\
\hline \multicolumn{2}{|l|}{ Uso del suelo } & \multicolumn{2}{|c|}{ Semi -intensivo } & Extensivo & \multicolumn{2}{|c|}{ Semi -extensivo } & Extensivo \\
\hline \multicolumn{2}{|l|}{ Carga media (cab/ha) } & 23,7 & 28,4 & 1,9 & 6,6 & 7,8 & 2,8 \\
\hline \multicolumn{2}{|c|}{ Superficie efectiva ovina (ha) } & 2,8 & 5,0 & 105,5 & 25,8 & 40,0 & 48,0 \\
\hline \multicolumn{2}{|l|}{ Praderas perennes (\%) } & 0,0 & 0,0 & 0,0 & 0,0 & 0,0 & 52,0 \\
\hline \multicolumn{2}{|c|}{ Verdeos invierno/verano (\%) } & 0,0 & 0,0 & 4,0 & 27,0 & 25,0 & 36,0 \\
\hline \multicolumn{2}{|l|}{ Rastrojos (\%) } & 0,0 & 0,0 & 95,0 & 72,0 & 75,0 & 0,0 \\
\hline \multicolumn{2}{|l|}{ Pastizal natural (\%) } & 100,0 & 100,0 & 1,0 & 0,0 & 0,0 & 23,0 \\
\hline \multicolumn{2}{|l|}{ Genética seleccionada } & $\begin{array}{l}\text { H. Down y } \\
\text { Criolla }\end{array}$ & $\begin{array}{l}\text { Corriedale y } \\
\text { Criolla } \\
\end{array}$ & $\begin{array}{l}\text { H. Down y } \\
\text { Corriedale }\end{array}$ & $\begin{array}{l}\text { Corriedale y } \\
\text { Pampinta }\end{array}$ & Corriedale & $\begin{array}{l}\text { H. Down y } \\
\text { Merino }\end{array}$ \\
\hline \multicolumn{2}{|l|}{ Suplementos } & $\begin{array}{l}\text { Fardos/maíz } \\
\text { Corderos }\end{array}$ & $\begin{array}{l}\text { Fardos/maíz } \\
\text { Corderos }\end{array}$ & $\begin{array}{l}\text { Fardos/maíz } \\
\text { Corderos }\end{array}$ & $\begin{array}{l}\text { Fardos/maíz } \\
\text { Hembras } \\
\text { gestantes }\end{array}$ & $\begin{array}{l}\text { Balanceado } \\
\text { Hembras } \\
\text { pre-servicio }\end{array}$ & $\begin{array}{c}\text { Balanceado, } \\
\text { maíz/rollos } \\
\text { Corderos } \\
\end{array}$ \\
\hline \multirow{2}{*}{$\begin{array}{l}\text { Comercialización } \\
\text { y destino de la } \\
\text { producción }\end{array}$} & 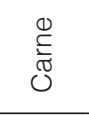 & $\begin{array}{l}\text { Restaurante } \\
\text { Circuito } \\
\text { turístico }\end{array}$ & $\begin{array}{l}\text { Venta local } \\
\text { Circuito } \\
\text { turístico }\end{array}$ & $\begin{array}{l}\text { Venta local y } \\
\text { restaurantes }\end{array}$ & Venta menı & ıdeo local & $\begin{array}{c}\text { Venta a } \\
\text { intermediarios } \\
\text { (frigoríficos) }\end{array}$ \\
\hline & 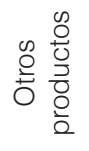 & $\begin{array}{l}\text { Guano, } \\
\text { venta local }\end{array}$ & Lana lavada & Lana clasifica & $\begin{array}{c}\text { da - Reproduc } \\
\text { zonal }\end{array}$ & tores, venta & $\begin{array}{c}\text { Reproductores } \\
\text { venta local y } \\
\text { regional -Lana } \\
\text { p/exportación }\end{array}$ \\
\hline \multicolumn{2}{|c|}{ Precio Corderos $(\$ / c a b)^{(1)}$} & 172 & 185 & 183 & 238 & 212 & 179 \\
\hline \multicolumn{2}{|l|}{ Precio Lana $(\$ / k g)^{(1)}$} & -- & 5,3 & 4,2 & 4,2 & 4,2 & 7,3 \\
\hline \multicolumn{2}{|c|}{ Precio Reproduct. (\$/cab) (1) } & -- & -- & 597 & 530 & 597 & 914 \\
\hline \multicolumn{2}{|l|}{ Financiamiento } & \multicolumn{6}{|c|}{ Propio y créditos de la Ley Ovina } \\
\hline
\end{tabular}

Fuente: Elaboración propia

Referencias: (1) Los valores monetarios se expresan en moneda constante de junio/2011.

Tabla 4. Indicadores físicos de los sistemas ovinos de Córdoba

\begin{tabular}{lcccccc}
\hline \multicolumn{1}{c}{ Indicadores físicos } & Caso 1 & Caso 2 & Caso 3 & Caso 4 & Caso 5 & Caso 6 \\
\hline Producción de carne (kg/oveja) & 13,0 & 1,0 & 10,1 & 3,41 & 40,3 & 47,3 \\
Producción de lana (kg/oveja) & 0,0 & 3,0 & 3,0 & 3,4 & 4,5 & 3,7 \\
Pérdidas (1)/Existencias (\%) & 33,0 & 63,0 & 30,1 & 23,0 & 8,0 & 14,0 \\
Señalada (\%) & 48,0 & 30,0 & 64,0 & 55,0 & 97,0 & 104,0 \\
Carneros (\%) & 2,0 & 2,0 & 3,0 & 3,0 & 5,0 & 4,0 \\
Consumo (2) (\%) & 0,0 & 137,0 & 8,0 & 9,0 & 3,0 & 5,0 \\
\hline
\end{tabular}

Fuente: Elaboración propia

Referencias: (1) mortandad, predadores y abigeato; (2) kg consumidos/kg producidos.

\section{Caso 5}

El sistema ovino del departamento Roque Sáenz Peña es gestionado por padre e hijo para el aprovechamiento de suelos inundables y salinos, y el beneficio que otorgó la línea de créditos de Ley 25.422 para la Recuperación de la Ganadería Ovina. Suplementó ovejas pre-servicio, manejo que demandó mayor control y mano de obra durante los nacimientos. A pesar de la diferencia de inventario de hacienda negativa $(-5 \%$ del IB), durante el ciclo se capitalizó en la categoría ovejas. De la entrevista surgió que proveedores ocasionales deprimen los precios del cordero, pues la actividad regional es para autoconsumo y sólo venden los 
excedentes. En alguna ocasión comercializaron con un frigorífico, pero a un precio 30\% menor que en la venta directa. Destacaron que la Ley Ovina debería abordar aspectos de comercialización de productos y subproductos, y señalaron la dificultad existente para conseguir personal idóneo en el manejo de los animales.

\section{Caso 6}

Ubicado en el departamento General Roca, posee una estructura productiva semejante a los sistemas ganaderos bovinos predominantes de la región (Guida Daza et al., 2011). Se distinguió de los restantes casos por constituir la base alimentaria con praderas perennes, que comparte con la ganadería bovina. También fue el único caso que comercializó corderos con intermediarios que operan en la región, y lana en el exterior, a través de una red cooperativa interprovincial.

\section{Método de análisis de los casos estudiados}

Para reforzar la validez del proceso de investigación se triangularon los resultados económicos obtenidos con los referenciados por otros investigadores, teniendo en cuenta las características ambientales y socio-económicas de cada región (Villareal Larrinaga y Landeta Rodríguez, 2009).

Iniciado el estudio se constataron las elevadas pérdidas por mortandad de reproductores y disminución productiva e incremento de costos de alimentación, ocasionados por una intensa sequía, que según el Instituto Meteorológico Nacional (MAGyP, 2011) comenzó en el norte de Córdoba en 2006 y se extendió a casi toda la provincia desde enero de 2007.

Para acrecentar la evidencia obtenida, en tres de los seis casos se determinaron los indicadores técnico-económicos para un segundo período. En este trabajo sólo se exponen los principales resultados obtenidos, mayores detalles fueron expuestos por Freire et al. (2010 y 2011b).

El análisis de los resultados económicos estimados se realizó mediante una simplificación de la propuesta de Caracciolo de Basco et al. (1981), la cual explica que éstos derivan de diversas estra- tegias productivas adoptadas por los productores. Las estrategias, a su vez, surgen de la interacción de variables estructurales e intervinientes (Figura 1).

Las variables estructurales (Tabla 2), conformadas por indicadores organizacionales como dotación y combinación de recursos productivos y la forma social del trabajo definen las condiciones de producción, las estrategias y los resultados (Caracciolo de Basco et al., 1981). Las variables intervinientes se refieren a aspectos culturales y actitudinales que también influyen en las estrategias adoptadas; entre otras se puntualizaron la forma de tenencia de la tierra, la existencia de ingresos extraprediales y las fuentes de información del productor.

Las estrategias productivas incluyen el análisis del uso del suelo, la tecnología empleada y la comercialización, entre otras (Tabla 3). Estas estrategias condujeron a resultados físicos y económicos que se exponen en las Tablas 4 y 5 y en las Figuras 2 a 5.

\section{RESULTADOSY DISCUSIÓN}

En primera instancia se presentan los principales resultados y análisis de los casos individuales. Las variables estructurales y las estrategias productivas se detallan, complementando el texto, en las Tablas 2 y 3. Para los sistemas evaluados en un segundo período se sintetiza la información con los resultados más significativos relacionados a su evolución. Por último, se realiza el análisis general de los casos, con discusiones individuales o conjuntas según el interés de particularizar o generalizar situaciones, establecer diferencias y similitudes.

\section{Análisis individual de los casos}

\section{Casos 1 y 2}

La calidad forrajera de los pastizales naturales de estos casos y la absoluta sequía ocurrida en la región diferenciaron la productividad del recurso tierra del monte árido, de los restantes casos de la región pampeana (Tsakougmagkos et al., 2000; MAGyP, 2011). En tal sentido, los capitales y la superficie efectiva destinados a la actividad ovina

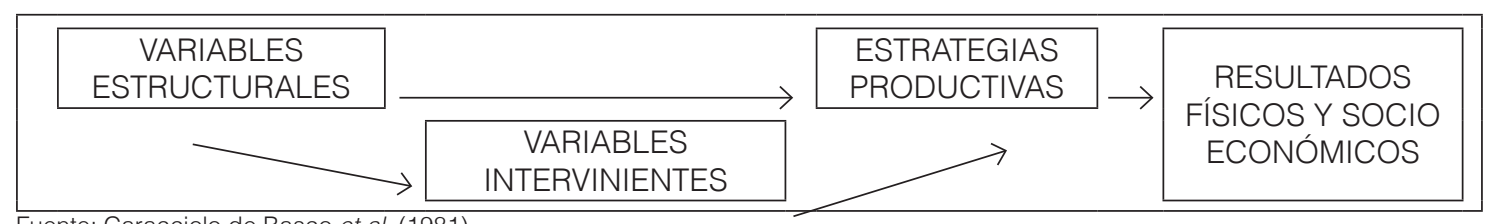

Fuente: Caracciolo de Basco et al. (1981)

Figura 1. Relación entre variables, estrategias y resultados productivos 
Tabla 5. Indicadores económicos de los sistemas ovinos de Córdoba

\begin{tabular}{lcccccc}
\hline \multicolumn{1}{c}{ Indicadores Económicos $^{(1)}$} & Caso 1 & Caso 2 & Caso 3 & Caso 4 & Caso 5 & Caso 6 \\
\hline Costo de mano de obra (\$/oveja) & 78,4 & 66,8 & 112,2 & 44,5 & 67,9 & 23,3 \\
Costo forrajes anuales (\$/oveja) & 0,0 & 0,0 & 12,0 & 19,6 & 8,1 & 45,7 \\
Costo suplementación (\$/oveja) & 25,0 & 20,2 & 3,3 & 4,2 & 45,0 & 79,1 \\
MB (\$/ha) & 1633,7 & 489,6 & 121,5 & 692 & 745,4 & 440 \\
MN (\$/ha) & $-289,4$ & $-1333,1$ & $-58,8$ & 488 & 269,8 & 283,9 \\
MB/CD (\$/\$) & 0,9 & 0,6 & 2,3 & 2,2 & 2,4 & 0,7 \\
MB/cordero faenado (\$/cab) & 76,2 & 153,0 & 388,4 & 247,5 & 161,2 & 293,3 \\
MN/CT $(\%)$ & $-7,8$ & $-49,2$ & $-17,0$ & 93,6 & 34,1 & 36,1 \\
\hline
\end{tabular}

Referencias: ${ }^{(1)}$ Los valores monetarios se expresan en moneda constante de junio/2011.

Fuente: Elaboración propia

se valoraron en ambos como "relativamente insuficientes" (Tabla 2). La escasa superficie ovina definió la intensividad de la actividad, con elevadas cargas medias (Tabla 3). La débil oferta forrajera y las pérdidas elevadas derivaron en reducidos índices productivos (Tabla 4).

En ambos prevalece la lógica productiva empresarial (Caracciolo de Basco et al., 1981). El caso 1 integra verticalmente la actividad ovina con su actividad comercial extra-agropecuaria, al llevar su producto "desde el campo a la mesa del consu-

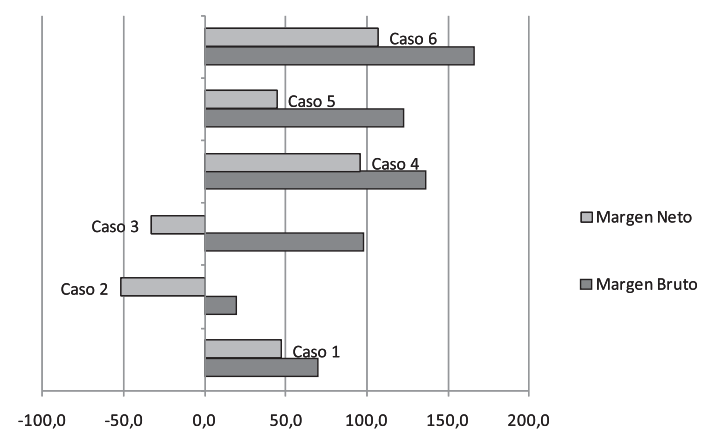

Fuente: Elaboración propia

Figura 2. Resultados económicos de los sistemas ovinos de Córdoba (\$/oveja)

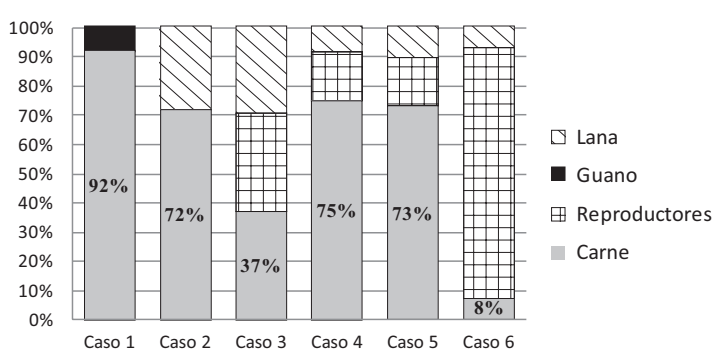

Fuente: Elaboración propia

Figura 3. Composición de ventas de los productos ovinos de Córdoba (\%) midor". Aún así, valoró el producto primario a costo de oportunidad de mercado, el menor precio de todos los analizados. El caso 2 sopesa continuar la actividad, de persistir las pérdidas físicas y económicas sufridas.

El caso 2 alcanzó el menor MB por oveja (Figura 2). En ambos casos, los MB positivos no alcanzaron a cubrir la totalidad de $\mathrm{Cl}$ y derivaron en $\mathrm{MN}$ negativos (Tabla 5). Los costos indirectos superaron a los directos (Figura 4). En los CT, mano de obra y esquila fueron los rubros de mayor incidencia para el caso 1, y mano de obra y suplementos para el caso 2 (Figura 5). Entre los sistemas con MN negativos, el caso 1 fue el menos ineficiente en el uso de los recursos (MN/CT): perdió 8 pesos cada 100 involucrados en la actividad (Tabla 5).

\section{Caso 3}

Sus recursos, totalmente amortizados, fueron los más reducidos entre los casos de la región pampeana, a excepción del capital fijo vivo que superó a los vientres de los casos 4 y 6 (Tabla 2). La forma de tenencia de la tierra diferenció a éste de todos los restantes. No disponer de tierra en propiedad, ni mejoras suficientes para la actividad ovina, generó cuellos de botella a nivel organizati-

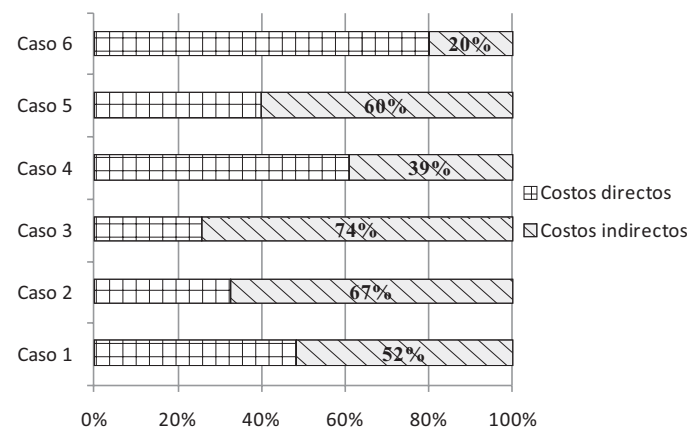

Fuente: Elaboración propia

Figura 4. Participación de costos de sistemas ovinos de Córdoba (\%) 


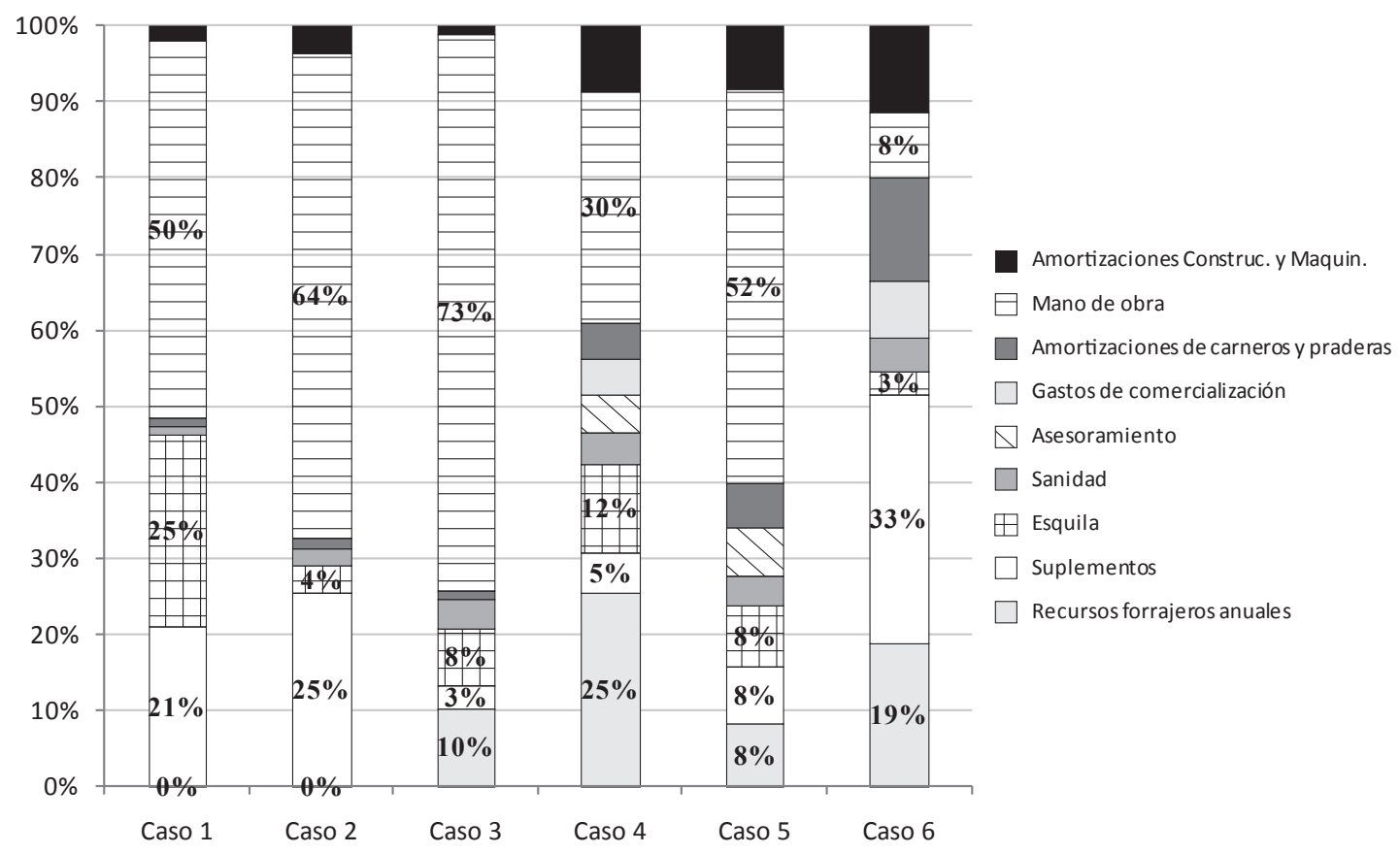

Fuente: Elaboración propia

Figura 5. Composición de costos totales de sistemas ovinos de Córdoba

vo y de costos, operando con elevada utilización de rastrojos (Tabla 3), mayores gastos de implantación de verdeos y escasa suplementación (Tabla 5). Con lógica productiva familiar y "ahorro en salarios", incurrió en elevados costos de oportunidad y baja eficiencia del factor trabajo dedicados a la actividad (Figura 5 y Tabla 5).

La escasa producción de carne produjo el menor $\mathrm{MB}$ de la región. Aunque fue uno de los más eficientes en la utilización de los recursos directos (MB/CD) y el de mayor retorno bruto por cordero faenado, su MN fue negativo (Tabla 5). Carne y reproductores participaron en proporciones similares en la composición del ingreso (Figura 3). Los costos indirectos superaron en más de un 300\% a los directos (Figura 4) debido a la mano de obra fundamentalmente (73\% de los CT); en importancia le siguieron forrajes anuales y esquila (Figura 5).

Durante el $2^{\circ}$ ciclo evaluado (2009/2010), el sistema incrementó en $24 \%$ la existencia media de ovejas y la mortalidad en $7 \%$ respecto al ciclo $2008 / 2009$, a la vez que disminuyeron los indicadores de señalada y la producción de carne en 18\% y $48 \%$, respectivamente. El MB -positivo- disminuyó, en tanto que el MN también fue negativo.

Caso 4

Semejante al caso 3 en la organización social del trabajo, se diferenció de aquel en la asignación de equivalentes hombre $(E H)$ a la actividad ovina (Tabla 2). Contaba con casi 100 ovejas, recurso tierra en propiedad y mayor valor de capitales -principalmente mejoras- que los casos 1 a 3; aunque de menor valor absoluto que los casos 5 y 6 . En términos relativos a los vientres, su capital superó al del caso 5 .

La comercialización asociativa con un grupo ovino de Cambio Rural -al cual asesora el productor-y su participación en el Programa Prolana le permitieron mejorar la calidad y precio de la lana (Tabla 3). Con elevada carga media, suplementación preservicio, y el mejor precio del cordero, obtuvo un destacado MB y el mayor MN (\$/ha) de todos los evaluados (Tabla 5). Fue el más eficiente en el uso de los recursos productivos totales (MN/CT) y casi triplicó al índice del caso 6. También fue uno de los más eficientes en el costo de mano de obra por oveja. Los CD superaron a los Cl, destacando mano de obra y verdeos en los CT (Figuras 4 y 5).

Este caso, también analizado en el ciclo 2009/2010, por razones de precio, rotación del capital invertido y pérdidas de reproductores, redujo la escala de la actividad ovina a favor de la producción porcina. Disminuyeron en 30\% las existencias de ovejas por muerte y ventas y, 75\% las de carneros, por ventas, muerte y abigeato. El porcentaje de señalada incrementó en 8 puntos y la producción de carne mejoró más de 200\%. El MB 
resultó un 10\% inferior respecto al del primer ciclo, aunque por disminución de los costos indirectos en 52\%, el MN no se modificó (Freire et al., 2011c).

Este comportamiento de cambio de escala a otra actividad más competitiva, estrategias adoptadas y resultados obtenidos parece indicar que esta producción familiar está desplazándose a la categoría empresarial, en un proceso de transición conocido como "diferenciación" de la racionalidad productiva (Piñeiro, 2000).

\section{Caso 5}

Posee superficie propia, importante capital en términos absolutos y relativos y el mayor número de vientres de todos los casos estudiados (Tabla 2). Con predominancia de mano de obra familiar e igual asignación de EH, logró mayor eficiencia en costos por oveja que el caso 3 (Tabla 5).

La racionalidad económica, semejante al caso 4, está en proceso de transición o diferenciación hacia la lógica productiva empresarial. En ambas unidades productivas, los hijos aportan capital cultural -estudios técnicos agropecuarios- a los sistemas, dado que sus actividades principales son extraprediales (Tabla 3). Mediante especialización y mejora genética proyecta establecer una cabaña, como objetivo de mediano plazo.

Con menores pérdidas y estrategias productivas -elevada carga media, genética, suplementación estratégica y mayor proporción de reproductoresobtuvo elevados indicadores físicos (Tablas 3 y 4), el mayor MB (\$/ha) y la mayor eficiencia económica (MB/CD) de todos los casos (Tabla 5). Los principales ingresos económicos se originaron con las ventas de carne y reproductores (Figura 3) y el mayor costo en el rubro mano de obra (Figura 5).

\section{Caso 6}

Con tierra propia e importante capitalización -corrales móviles, galpón de esquila, esquiladora, trailer y la proporción imputada de una camioneta-, adecuado manejo técnico y uso racional de los recursos, obtuvo los mayores indicadores de señalada y de producción de carne (Tablas 3 y 4), a expensas de elevados gastos de suplementación y alimentación forrajera anual. La Figura 2 lo destaca como el caso más eficiente respecto al capital hacienda (MB y MN). Sus CD superaron a los Cl (Figura 4).

Evaluado también en un segundo período, 2010/2011, este sistema incrementó un 5\% el número de madres y mantuvo la participación de ventas relativamente estable. El IB incrementó un 57\%, debido fundamentalmente al cambio de valor de la diferencia de inventario $(-16,3 \%$ a $20,7 \%)$. Con menores requerimientos de suplementación a causa de la mejora climática, se destacaron los rubros "implantación de verdeos" (45,8\%) y "amortizaciones de praderas y reproductores" (19,8\%) en los CD. La actividad ovina proporcionó ganancias en ambos ciclos, e incrementó su MN, triplicando al del ciclo anterior (Freire et al., 2011b).

\section{Análisis general de los casos}

En los casos evaluados las majadas no superaron las 250 cabezas/EAP, y la actividad ovina, con participación secundaria o complementaria, se orientó fundamentalmente hacia la producción de carne, ratificando los resultados obtenidos con la información de referencia (Mc Cormick, 2003; Rodríguez et al. 2010; MAGyP, 2011). Si bien la mayoría de los sistemas obtiene dos o más productos ovinos, los mayores ingresos se originaron en la venta de carne de cordero ( $83 \%$ de los casos). Coincidiendo con lo expuesto por Agüero et al. (2010), la carne de cordero se comercializó a través de la venta directa en cinco de los seis casos estudiados.

Los márgenes brutos estimados para las diferentes agro-regiones de Córdoba fueron positivos, tanto en establecimientos serranos como de llanura, extensivos o intensivos, especializados en carne o diversificados, coincidiendo con lo señalado por diferentes autores (Freire et al. 2009, 2010, 2011b; Issaly et al. 2010; Rodríguez et al, 2010; Arzubi et al, 2011, 2012).

En la composición de costos prevalecieron los rubros "mano de obra", "suplementos", "forrajes anuales" y "esquila" con participaciones medias de $41 \%, 17 \%, 12 \%$ y $8 \%$. Los valores máximos fueron $73 \%, 33 \%, 25 \%$ y $25 \%$, y los mínimos 18\%, 3\%, $8 \%$ y $3 \%$, respectivamente.

La sequía del ciclo se manifestó con mayor intensidad en las regiones semiárida norte, serrana del oeste y semiárida del centro oeste -casos 1 a 3-, originando exiguos parámetros productivos y económicos. Guida Daza et al. (2011) determinaron también resultados netos negativos en los ciclos 2007/2008 a 2009/2010 para los sistemas productivos representativos de esas $\mathrm{ZAH}$.

Como lo expresaron Freire et al. (2011a), factores adicionales al clima y al ambiente ocasionaron mayor vulnerabilidad en estos EAP: pequeña escala y elevados costos en el caso 1, y costosa suplementación y elevadas pérdidas por depredadores naturales en el caso 2. Al respecto, en IDIAXXI (2004) se vincularon disminuciones de rentabilidad y de patrimonio con pérdidas cercanas a un $20 \%$. Este valor de referencia fue ampliamente superado en los casos 1 a 3, durante el ciclo de sequía. Los autores también consideraron productivamente inadecuado obtener porcentajes de señalada inferiores al $65 \%$, 
valor que sólo superaron los casos 5 y 6 .

En el sistema 3, además de las pérdidas sufridas, se apreciaron problemas de organización de los recursos, coincidiendo con los hallazgos de Mc Cormick (2003). El crecimiento de existencias y la disminución de indicadores físicos y económicos entre los ciclos analizados, refuerza la hipótesis de una majada sobredimensionada en relación a los recursos restantes; fundamentalmente la reducida disponibilidad de capital circulante para remunerar mano de obra y afrontar gastos de alimentación y suplementación, mayores en situación de sequía. Rodríguez et al. (2010) señalaron que mejorar la tasa de señalada tiene mayor impacto económico que incrementar la escala, dada la mayor inversión requerida.

En línea con las expresiones de Rodríguez et al. (2011), los casos 4 a 6 de la región pampeana, centro este, sureste y sur provincial, cubrieron los costos, generaron excedentes y contribuyeron a la estabilidad de los EAP. En moneda de junio/2011, los MB ovinos fueron semejantes o superiores a los MB de invernada vacuna $(557,3$ \$/ha) de la región centro de Córdoba (Agromercado, 2009). Coincidiendo con lo expresado por Santamaría et al. (1998), estos sistemas basaron la alimentación sobre bases forrajeras implantadas y lograron los mejores parámetros productivos y económicos. En semejanza con las expresiones de Vigliocco et al. (2011), los casos 4 y 5 aprovecharon eficientemente los rastrojos agrícolas y obtuvieron mayores $\mathrm{MB}$ por hectárea que el caso 6 , con menores costos de forrajes anuales y suplementación.

Se destacaron dos casos con larga tradición ovina, diferentes estrategias y recursos productivos. Un pequeño sistema familiar capitalizado obtuvo el mayor margen neto en \$/ha; el otro, empresarial, logró el mayor margen neto en \$/oveja. Ambos coincidieron en la organización de los recursos al asignar mayores capitales a la actividad y reducida utilización del factor trabajo.

Si bien todos los casos se beneficiaron con créditos otorgados por la Ley Ovina, en algunas entrevistas se manifestó la necesidad de contar con instrumentos que permitan una mayor articulación comercial entre la producción primaria y la industria, como también la posibilidad de acceder oportunamente a créditos flexibles, frente a situaciones climáticas extremas.

Se concluye que la producción ovina permitió acrecentar eficazmente el beneficio económico en sistemas que contaban con recursos suficientes. En casos con problemas endógenos como escala, organización o financiamiento, sumados a condiciones exógenas adversas de clima, ambiente, abigeato o mercado, entre otros, no alcanzaron a cubrir los costos de producción, por lo que de no mediar cambios de estrategias y de políticas públicas que afectan al sector, su viabilidad de mediano o largo plazo se verá afectada.

\section{AGRADECIMIENTOS}

Agradecemos la predisposición de los productores que aportaron sus datos y su mirada respecto a las oportunidades y problemáticas que enfrentan en el desarrollo de esta actividad.

\section{BIBLIOGRAFÍA}

Agüero, D.; V. Freire, M. Peralta, M. Vigliocco y G. Sandoval, 2010. Diagnóstico de la Cadena Ovina en la Provincia de Córdoba. Argentina. Revista Mexicana de Agronegocios: Quinta Época. Año XIV. 26: 161-177.

Agromercado, 2009. Márgenes de invernada, centro de Córdoba. Revistas Año 28, º290, p. 79 y Año 29, Nº 293, p. 73.

Arzubi, A.; M. Mc Cormick, G. Lynch y L. Simonetti, 2011. Evaluación técnico-económica de la producción ovina considerando los sistemas de producción más difundidos en cada Región. $3^{\circ}$ Congreso Regional de Economía Agraria. Valdivia, Chile, pp. 142:143.

Arzubi, A.; G. Lynch, M. Mc Cormick y L. Simonetti, 2012. Análisis de modelos representativos y mejorados de la producción ovina desarrollada en regiones de Buenos Aires y Mesopotamia. XLIII Reunión Anual Asoc. Argentina de Economía Agraria (AAEA). Corrientes, pp. 9 -11.

Caracciolo de Basco, M.; P. Tsakoumagkos, C. Rodríguez Sánchez y M. C. Borro, 1981. Esquema conceptual y metodología para el estudio de tipos de establecimientos agropecuarios con énfasis en el minifundio. Secretaría de Agricultura y Ganadería de la Nación. Servicio Nacional de Economía y Sociología Rural. Grupo de Sociología Rural. Buenos Aires, 77 pp.

Frank, G. 1985. Introducción al cálculo de costos agropecuarios. $5^{\circ}$ Ed. El Ateneo. Bs. As. 37 pp.

Freire, V.; D. Agüero, M. Peralta, J. Salminis, G. Sandoval, M. Vigliocco, R. Martinez, M. Caviagliasso y J. Sánchez, 2009. Resultados económicos de sistemas productivos con ganadería ovina en la provincia de Córdoba. Estudio de casos. Comunicación (digital). XL Reunión Anual de AAEA. Bahía Blanca, Buenos Aires, 8 pp.

Freire, V.; M. Vigliocco, D. Agüero, M. Peralta, M. Caviagliasso y G. Sandoval, 2010. Resultados económicos de un sistema productivo ovino de la región centro-este de Córdoba. XLI Reunion Anual de Economia Agraria. San Luis. Comunicación, 8 pp. 
Freire, V.; D. Agüero, M. Vigliocco, G. Sandoval, M. Ponce Crivellaro, M. Peralta, J. Salminis y M. Caviagliasso. 2011a. Análisis económicos de sistemas productivos ovinos de Córdoba, Argentina. $3^{\circ}$ Congr. Reg. de Economía Agraria. Valdivia, Chile. Comunicación, p. 167.

Freire, V.; M. Ponce Crivellaro, D. Agüero, M. Peralta, G. Sandoval, M. Vigliocco, J. Roberi, M. Vigliocco y J. Sánchez, 2011b. Indicadores económicos de un sistema ovino de Córdoba (Argentina). Revista Argentina de Producción Animal Vol 31 Supl 1, p. 238.

Freire, V.; M. Vigliocco, M. Ponce Crivellaro, G. Sandoval, D. Agüero, M. Caviagliasso, M. Peralta y J. Salminis, 2011c. Evaluación económica de un sistema mixto con actividad ovina del centro este de Córdoba. V Jornadas Científico-Técnicas, FAV, UNRC, 4 pp.

Ghida Daza, C. y C. Sánchez, 2009. Estudios socioeconómicos de la sustentabilidad de los sistemas de producción y recursos naturales Nㅜ10. Zonas Agroeconómicas Homogéneas. Córdoba, 263 pp. <http://ZAH_INTA_Cba_2009.pdf> Consultada el 28/09/2010.

Ghida Daza, C.; C. Sánchez, C. Issaly, L. Pizarro (Coordinadores), V. Freire, G. Gigena, M. Llop, M. Peralta, M. Ponce Crivellaro, D. Reynoso, J. Salminis, B. Urquiza y M. Vigliocco, 2011. Monitoreo económico de los sistemas productivos predominantes del sector agropecuario de Córdoba. Resultados de las campañas 2006/07, 2008/09 y 2009/10 y 2010/11. Vol X. Convenio INTA/ MAGyA Cordoba/ UNRC, Editorial UNRC, 53 pp.

IDIAXXI, 2004. Diagnóstico de la Producción Ovina de Corrientes. Comisión Mixta Provincial del Ovino Provincia de Corrientes. INTA Buenos Aires, pp. 2733. <http://IDIA_7.pdf.> Consultada el 21/09/2011.

INDEC, 2012. <http://www.indec.gov.ar> Consultada el $12 / 09 / 2011$.

Issaly, L.; A. Decara, M. Peralta, M. Vigliocco y A. Sandoval, 2010. Estrategias de comercialización de pequeños y medianos productores de carne ovina y caprina en el sur de la provincia de Córdoba- Argentina: estudios de casos. Cuad. de Desarrollo Rural 7, 65: 87-109.

MAGyP, 2011. Anuario 2010. Ganados y carnes. Cuestas Acosta, F. (compiladora) y A. Lotti (director). Subsecretaría de Ganadería. SAGyP. $1^{\text {a }}$ ed. - Buenos Aires <http://64.76.123.202/site/ganaderia/anuario/pdf/ANUARIO\%202010\%20COMPLETO\%20web.pdf> Consultada el 09/02/2011.

Mc Cormick, M., 2003. Problemática ovina en Argentina: Un análisis de los esquemas de gestión. Cuadernos de CEAgro 4:35-43.

Peretti, M.; C. Issaly,L. Pizarro (Coordinadores), V. Freire,
C. Ghida Daza, A. Prado, J. Salminis, C. Sánchez, B. Urquiza y M. Vigliocco, 2006. Monitoreo económico de los sistemas productivos predominantes del sector agropecuario de Córdoba. Resultados de 2003/04, 2004/05 y 2005/06 - Vol IX, Convenio INTA/SAGyRR Cordoba/ UNRC, Ed. UNRC, 78 pp.

Piñeiro, D., 2000. Caracterización de la producción familiar. <http://www.fagro.edu.uy/ suinos/biblioteca/ paf/Pineiro_.pdf> Consultada el 18/02/13.

Revista Agromercado. 2009. Año 28. № 291 y 292. Modelo de Márgenes de Invernada.

Rodríguez, C.; E. Ponssa y D. Sánchez Abrego, 2010. Evaluacion económica y productiva de modelos de produccion ovina en la provincia de Buenos Aires. XLI Reunión Anual de AAEA. Potrero de los Funes, San Luis, $18 \mathrm{pp}$.

Rodríguez, M.; J. Pueyo y J. Dupleich, 2011. Sistema ganadero de cría vacuna mixto con ovinos. Modelización de sistemas de producción de Entre Ríos: Estudio económico y de riesgo, EEA Paraná. Serie de Extensión N 63. INTA, Centro Regional Entre Ríos. pp 77-87.

Santamaría, C.; I. Gárriz, A. Rodríguez, C. Díez De Ulzurrun y J. Ochoa, 1998. Ovino de carne: Resultados económicos de diferentes sistemas de producción. Producción Ovina y Caprina. XXIII Jorn. Científ. Soc. Española de Ovinotec. y Caprinotec. (SEOC), pp. 259-263.

SENASA. 2008. Sistema de Gestión Sanitaria. Disponible en: <http://www.senasa.gov.ar/index.php> Consultada el 21/02/08.

Tsakougmagkos, P.; S. Soverna y C. Craviotti, 2000. Campesinos y pequeños productores en las regiones agroeconómicas de Argentina. Proinder. Serie de Doc. 2. Bs. As. 62 pp.

Vigliocco, M.; V. Freire, D. Agüero, M. Peralta, G. Sandoval, M. Ponce Crivellaro, J. Sánchez, R. Martinez, M. Cavigliasso y J. Salminis, 2011. Vientos favorables para la carne de cordero. Revista Prisma, Año 3 № 5. Dossier de Agronomía de la Revista PRISMA.

Villareal Larrinaga, O. y O. Landeta Rodríguez, 2009. El estudio de casos como metodología investigación científica en dirección y economía de la empresa. Una aplicación a la internacionalización. Investigaciones Europeas de Dirección y Economía de la Empresa. Vol. 16, № 3, 2010, pp. 31-52.

Yin, R. K. 1994. Case Study Research. Design and Methods in MILES, M and M. HUBERMAN. 1994. Qualitative Data Analysis. $2^{\circ}$ Ed. Thousand Oaks, CA: Sage Publications. 